\title{
Hyper Raman Scattering in Microcavity Quantum Wells: A Quantum Optical Process in the Strong Coupling Regime
}

\author{
S. Savasta, R. Girlanda, and G. Martino \\ Istituto Nazionale per la Fisica della Materia and Università di Messina, \\ Dipartimento di Fisica della Materia e Tecnologie Fisiche Avanzate, Salita Sperone 31, \\ I-98166 Sant'Agata-Messina, Italy
}

\begin{abstract}
We analyze the hyper Raman scattering process in a quantum well grown inside a semiconductor microcavity and present a microscopic calculation of emission spectra as a function of excitation energy, detuning, and angles. The exciton-photon coupling is treated non-perturbatively to include polariton effects and the Coulomb interaction between electrons is treated beyond the mean field approximation. The observation of hyper Raman scattering in semiconductor microcavities would represent an important step for the realization of non-classical optical states based on excitons. We find that the polaritons emitted in this scattering process are strongly correlated. This correlation, can be efficiently transferred to output photons, resulting in emission of two correlated non-classical light beams. As a probe of the quantum correlation we calculate the output spectrum of fluctuations in the intensity difference of the two beams generated in the hyper Raman process.
\end{abstract}

The atom-cavity system has been used to investigate quantum dynamical processes for open quantum systems in a regime of strong coupling and to explore quantum optical effects [1]. By quantum optical effects we mean those effects which are intimately tied to the quantization of the optical field. This distinguishes them from effects that can be described by a semiclassical theory, where only the variables of the material system are quantized. With the advent of high-quality microcavity Quantum Wells (QW's) it is possible to observe cavity quantum electrodynamics effects in solid state systems [2, 3]. The question arises if it is possible to observe quantum optical effects in exciton systems or if the strong absorption and relaxation processes, proper of these systems, prevent the observation of non-classical subtle effects. The rather surprising answer to this question is that a quantum optical effect in bulk excitonic polaritons was yet observed in 1978 by Phach et al. [4] and by Hönerlage et al. [5]. They determined the polariton dispersion in $\mathrm{CuCl}$ by resonant Hyper Raman Scattering (HRS) via biexcitons. Hyper Raman scattering (or two-photon Raman scattering) is a well known efficient technique of nonlinear optical spectroscopy to determine the bulk polariton dispersion. HRS can be schematically described as follows: two incident photons of given energy and wave vectors propagate inside the crystal as polaritons and create a virtual two electron-hole $(\mathrm{e}-\mathrm{h})$ pair state which acts as intermediate state to create two final polaritons or a longitudinal exciton and a polariton. The emission from final polaritons can be detected experimentally. Energy and momentum have to be conserved in the whole process. If the crystal is excited by a laser beam of given photon energy $\hbar \omega_{i}$, one finds

$$
\begin{aligned}
& \hbar \omega_{s}+\hbar \omega^{\prime}=2 \hbar \omega_{i}, \\
& \mathbf{k}_{s}+\mathbf{k}^{\prime}=2 \mathbf{k}_{i},
\end{aligned}
$$


where $\hbar \omega_{s}$ and $\mathbf{k}_{s}$ are the energy and momentum of the detected final polariton, while $\hbar \omega^{\prime}$ and $\mathbf{k}^{\prime}$ are the energy and momentum of the longitudinal exciton or the other polariton. Of course polariton energies and momenta inside the crystal are not independent but are related by the frequency dependent dielectric function $c^{2} k^{2} / \omega^{2}=\varepsilon(\mathbf{k})$. The determination of the polariton dispersion has successfully been accomplished by HRS in several large gap bulk semiconductors [6]. Hyper Raman scattering, being a nonlinear coherent process related to the third-order nonlinear susceptibility, can be considered as a spontaneous non-degenerate four wave mixing. In Four Wave Mixing (FWM) the optical decay of the virtually excited biexcitons is stimulated by sending an additional light beam, while in HRS the decay is determined by intrinsic quantum fluctuations. The spontaneous optical decay of the two e-h pair state cannot be described in the framework of a semiclassical theory. In this respect HRS is a process intimately tied to the quantization of the optical field [7]. So we stress on the fact that HRS has to be regarded not only as an efficient technique for the investigation of polariton dispersion but also as an efficient tool to explore excitonic quantum dynamics. Furthermore, at our knowledge, HRS is the only manifestation of excitonic quantum optical dynamics observed experimentally. The hyper Raman forward scattering spectra in $\mathrm{CuCl}$ [4] and their incident angle dependence are well described by a microscopic theory of HRS in bulk semiconductors [8] based on a general theory of the nonlinear optical response of an exciton system, which does not rely on the semiclassical factorization [7].

In this paper we analyze the hyper Raman scattering process in one or more QWs grown inside a semiconductor microcavity. We present a microscopic calculation of emission spectra as a function of excitation energy, detuning and angles. Furthermore we analyze the quantum correlations which can be induced by this nonlinear scattering process.

We start from the Hamiltonian $H_{\mathrm{s}}$ of the usual semiconductor model $[9,10]$. The states $\left|E_{N, \alpha, \mathbf{k}}\right\rangle$ with energy $\omega_{N, \alpha, \mathbf{k}}$ of $H_{\mathrm{S}}$ can be labelled according to the number $N$ of $\mathrm{e}-\mathrm{h}$ pairs and the total momentum $\mathbf{k}[9,10,7]$. The state $\left|E_{N=0}\right\rangle$ is the semiconductor ground state. The $N=1$ subspace is the exciton subspace with the additional quantum number $\alpha=(n, \sigma)$ that label exciton states [10], being $n$ the exciton level and $\sigma$ the polarization. The set of states with $N=2 \mathrm{e}-\mathrm{h}$ pairs determines the biexciton subspace. In the following, for the sake of simplicity we will omit polarization labels. The interaction of the electron system with cavity modes is given in the usual rotating wave approximation by

$$
H_{\mathrm{I}}=i \hbar \sum_{n, \mathbf{k}} V_{n, \mathbf{k}} a_{\mathbf{k}}^{\dagger} B_{n, \mathbf{k}}+\text { h.c. },
$$

where the operator $a_{\mathbf{k}}^{\dagger}$ creates a cavity photon of given in-plane wave vector $\mathbf{k}$ with energy $\omega_{\mathrm{c}, \mathbf{k}}=\left(\omega_{0}^{2}+v^{2} \mathbf{k}^{2}\right)^{1 / 2}$, being $v$ the velocity of light inside the cavity, $B_{n, \mathbf{k}}^{\dagger}$ creates an exciton state with the same in-plane wave vector $\mathbf{k}$ and energy $\omega_{1, n, \mathbf{k}} \cdot V_{n, \mathbf{k}}$ is the photon-exciton coupling coefficient. Following [10,7] we introduce the Hubbard operators $\hat{X}_{N, \alpha, \mathbf{k} ; M, \beta, \mathbf{k}^{\prime}}=\left|E_{N, \alpha, \mathbf{k}}\right\rangle\left\langle E_{M, \beta, \mathbf{k}^{\prime}}\right|$ which can be used to express the exciton operators. In the following we will write the coupled exciton-photon equations of motion restricting the space of the electronic system up to states with $N=2 \mathrm{e}-\mathrm{h}$ pairs, which is sufficient when describing processes up to the lowest nonlinear order. This is justified by a truncation procedure [7] which is the generalization to the full quantum case of a similar procedure introduced by Axt and Stahl [11], and Victor et al. [12] in the semi- 
classical case. The truncation scheme is analogous to the classification of the nonlinear optical processes [13]. The Coulomb potential is exactly taken into account in this truncation scheme [10], thus this procedure allows to go over the random-phase approximation in which the Coulomb correlations are factorized and semiconductor Bloch equations are obtained. In our case the perturbative parameter is the coherent laser field $\mathcal{E}$ pumping the cavity modes. By using the Hubbard operators we write the coupled Heisenberg-Langevin equations for the exciton and photon operators. We obtain

$$
\begin{aligned}
& \frac{\partial}{\partial t} a_{\mathbf{k}}=-\left(i \omega_{\mathrm{c}, \mathbf{k}}+\gamma_{\mathrm{c}}\right) a_{\mathbf{k}}+\sum_{n} V_{n, \mathbf{k}} B_{n, \mathbf{k}}+\mathcal{E}_{k}+\hat{F}_{\mathrm{c}, \mathbf{k}}(t) \\
& \frac{\partial}{\partial t} B_{n, \mathbf{k}}(t)=-\left(i \omega_{1, n, \mathbf{k}}+\gamma_{\mathrm{x}}\right) B_{n, \mathbf{k}}(t)-V_{n, \mathbf{k}} \hat{a}_{\mathbf{k}}(t) \\
&+\sum_{n^{\prime}, \mathbf{k}^{\prime}} \hat{X}_{1, n^{\prime}, \mathbf{k}^{\prime}-\mathbf{k}}^{\dagger}(t) \hat{R}_{n, n^{\prime}, \mathbf{k}^{\prime}}^{(2)}(t)+\hat{F}_{n, \mathbf{k}}(t),
\end{aligned}
$$

where $\hat{R}_{n, n^{\prime}, \mathbf{k}^{\prime}}^{(2)}(t)$ is given by

$$
\begin{aligned}
\hat{R}_{n, n^{\prime}, \mathbf{k}^{\prime}}^{(2)}(t)= & \sum_{n^{\prime \prime}, \mathbf{k}^{\prime \prime}} \Omega_{n, n^{\prime}, n^{\prime \prime}}^{(1)} \hat{X}_{0 ; 1, n^{\prime \prime}, \mathbf{k}^{\prime \prime}+\mathbf{k}^{\prime} / 2}(t) \hat{a}_{-\mathbf{k}^{\prime \prime}+\mathbf{k}^{\prime} / 2}(t) \\
& -i \sum_{\beta} c_{n, n^{\prime}, \beta}^{(1)} \hat{X}_{0 ; 2, \beta, \mathbf{k}^{\prime}}(t)
\end{aligned}
$$

The coefficients $\Omega_{n, n^{\prime}, n^{\prime \prime}}^{(1)}$ and $c_{n, n^{\prime}, \beta}^{(1)}$, whose $\mathbf{k}$ dependence has been dropped for sake of simplicity, are given in [7]. The summation in $(3 \mathrm{~b})$ is the nonlinear source term for the interband polarization. Nonlinear optical effects [10] as well as quantum optical correlations [7] origins from $R^{(2)}$. The first summation in (4) is the phase space filling term, while the second summation comes from Coulomb interactions between electrons. We have also considered in Eq. (3b) the exciton dephasing due to degrees of freedom not included (e.g. phonons) by adding a phenomenological term $\gamma_{\mathrm{x}}$ which leads to damping. The linear coupling of cavity modes with the external modes provides both the damping $\gamma_{\mathrm{c}}$ and the input optical pumping $\mathcal{E}_{k}$ of cavity modes. Together with the damping terms also the Langevin operators $\hat{F}_{\mathrm{c}, \mathbf{k}}(t)$ and $\hat{F}_{n, \mathbf{k}}(t)$ have to be introduced [14]. They are quantum noise operators with zero expectation values which maintain the photon and exciton commutation rules $[14,15]$ and have a variance different from zero. We notice that the operator equation $(3 \mathrm{~b})$ has the same structure of the coresponding equation of the semiclassical theory [10] applied to analyze the FWM response. FWM in semiconductors is essentially a semiclassical process. The source term of FWM can be identified with the following expectation value [10]:

$$
\sum_{n^{\prime}}\left\langle\hat{X}_{0 ; 1, n^{\prime}, \mathbf{k}^{\prime}}^{\dagger}\right\rangle^{(1)}\left\langle\hat{R}_{n, n^{\prime}, 2 \mathbf{k}_{i}}^{(2)}\right\rangle^{(2)}
$$

where the superscript labels the perturbative order. The incident light beam at $\mathbf{k}_{i}$ creates a nonlinear grating, which is described by $\left\langle\hat{R}_{n, n^{\prime}, 2 \mathbf{k}_{i}}^{(2)}\right\rangle$. An additional probe light beam at $\mathbf{k}^{\prime}$ creates a polarization $\left\langle\hat{X}_{0 ; 1, n^{\prime}, \mathbf{k}^{\prime}}\right\rangle^{(1)}$ with the same wave vector which is diffracted by the grating giving rise to signal at $\mathbf{k}_{s}=2 \mathbf{k}_{i}-\mathbf{k}^{\prime}$. In HRS there is no additional light beam and $\left\langle\hat{X}_{1, n^{\prime}, \mathbf{k}^{\prime}}\right\rangle=0$. However, although the mean is zero, its variance is not. This is due to the quantum mechanical zero point motion of excitons. In this case we can say that it is the exciton system which probes itself, and it is the quantum 
mechanical zero point motion of excitons that is diffracted by the grating. Since also in HRS as in FWM the grating is driven by the coherent laser field we can replace in Eq. (3b) the operator $\hat{R}^{(2)}$ by the c-number $R^{(2)}$ corresponding to its semiclassical expectation value. With this replacement we can easily solve Eq. (3). We consider a monochromatic continuous wave pump field $\mathcal{E}_{\mathbf{k}_{i}}$ at energy $\omega_{i}$ and calculate the steady state spectrum of the emitted photons which is given by

$$
I_{\mathrm{HRS}}\left(\omega_{s}\right)=2 \gamma_{\mathrm{out}} \int_{-\infty}^{+\infty} \mathrm{d} \tau \mathrm{e}^{i \omega_{s} \tau}\left\langle a_{\mathbf{k}_{s}}^{\dagger}(t) a_{\mathbf{k}_{s}}(t+\tau)\right\rangle_{\mathrm{ss}},
$$

where $\gamma_{\text {out }}$ is the loss coefficient of the output mirror, and $\mathbf{k}_{s}$ is determined by $\omega_{s}$ and by the observation angle $\theta_{\text {out }}, k_{s}=\omega_{s} \sin \theta_{\text {out }} / c$. Considering only the resonant 1 s heavy hole exciton level, we obtain

$$
\begin{aligned}
I_{\mathrm{HRS}}\left(\omega_{s}\right)= & \gamma_{\text {out }}\left|R_{2 \mathbf{k}_{i}}^{(2)}\left(2 \omega_{i}\right)\right|^{2} V_{1 \mathrm{~s}}^{2}\left(2 \gamma_{\mathrm{c}} V_{1 \mathrm{~s}}^{2}+2 \gamma_{\mathrm{x}}\left|i \omega_{\mathrm{c}, \mathbf{k}^{\prime}}-i \omega^{\prime}+\gamma_{\mathrm{c}}\right|^{2}\right) \\
& \times\left|G\left(\mathbf{k}^{\prime}, \omega^{\prime}\right) G\left(\mathbf{k}_{s}, \omega_{s}\right)\right|^{2},
\end{aligned}
$$

where $G(\mathbf{k}, \omega)=\left[\left(i \omega_{1,1 \mathrm{~s}, \mathbf{k}}-i \omega+\gamma_{\mathrm{x}}\right)\left(i \omega_{\mathrm{c}, \mathbf{k}}-i \omega+\gamma_{\mathrm{c}}\right)+V_{1 \mathrm{~s}}^{2}\right]^{-1}$ is the polariton propagator, $\omega^{\prime}=2 \omega_{i}-\omega_{s}$, and $\mathbf{k}^{\prime}=2 \mathbf{k}_{i}-\mathbf{k}_{s}$. In Fig. 1 we plot two HRS spectra in an almost normal configuration both for the pump and the observation direction. We calculate the emission spectra for a typical high quality microcavity by using $V_{1 \mathrm{~s}}=5.6 \mathrm{meV}$ for the exciton-photon coupling, $\gamma_{\mathrm{x}}=1 \mathrm{meV}$ and $\gamma_{\mathrm{c}}=0.7 \mathrm{meV}$ for the exciton and cavity broadenings. The cavity mode at $\mathbf{k}=0$ is resonant with the bare exciton energy $\hbar \omega_{1,1 \mathrm{~s}, 0}=1.4865 \mathrm{eV}$ in one spectrum, while it is slightly detuned $\Delta=6 \mathrm{meV}$ in the other. We choose the pump energy in between the Rabi peaks $2 \hbar \omega_{i}=\hbar \omega_{1,1 \mathrm{~s}, 0}+\hbar \omega_{\mathrm{c}, 0}$. We notice that the spectra are narrowed with respect to transmission or reflectivity spectra as in cavity resonance fluorescence [16], due to the factor $\left|G\left(\mathbf{k}^{\prime}, 2 \omega_{i}-\omega_{s}\right) G\left(\mathbf{k}_{s}, \omega_{s}\right)\right|^{2}$ in Eq. (7) originating from the quantum scattering process.

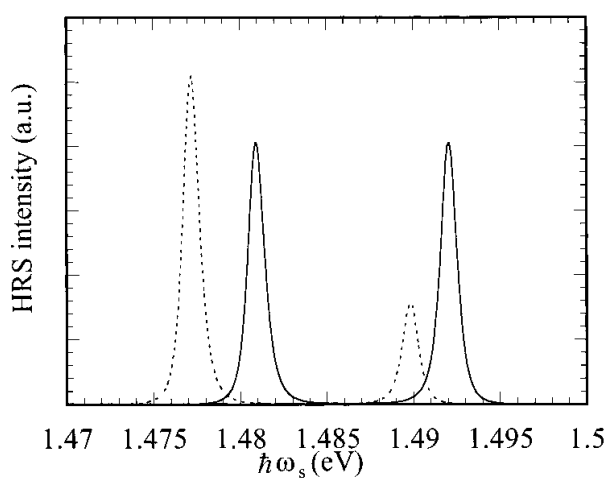

Fig. 1

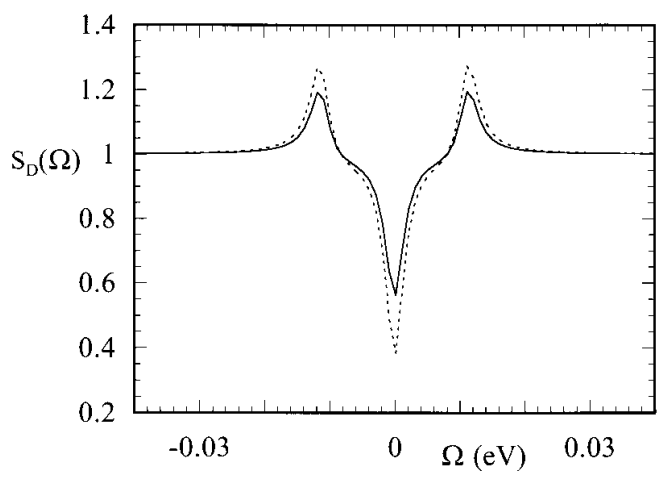

Fig. 2

Fig. 1. HRS spectra in an almost normal configuration both for the pump and the observation direction, for zero detuning (full line) and for a detuning $\Delta=6 \mathrm{meV}$ (dashed line). The parameters are given in the text

Fig. 2. Spectrum of fluctuations in the intensity difference of the two beams generated in the HRS process $S_{\mathrm{D}}(\Omega)$ for the same configuration and parameters of Fig. 1 (full line). The dashed line curve differs from the full one only for the choice of the broadenings $\left(\gamma_{\mathrm{x}}=0.7 \mathrm{meV}\right.$ and $\left.\gamma_{\mathrm{c}}=1 \mathrm{meV}\right)$ 
This should allow to separate the coherent signal from broader luminescence. We also notice that, in the presence of detuning, the peak at lower energy in Fig. 1, corresponding to a photon-like polariton, is more intense than the second peak, which correspond to an exciton-like polariton. This can be explained on the basis of the scattering process. Due to energy conservation, the "probe" which determines the first peak is the excitonic zero point motion at the energy of the second peak and vice versa. Thus being the excitonic zero point motion larger for an exciton-like polariton rather than a photon-like one, the first peak origins from the more intense probe.

The scattering process produces two polaritons with wave vectors $\mathbf{k}_{s}$ (model 1) and $\mathbf{k}^{\prime}$ (mode 2), obeying Eq. (1), which are expected to be strongly correlated. As a consequence the intensity difference of the two beams will carry fluctuations below shot noise. By using the solution of Eq. (3) we calculate the normalized spectrum of fluctuations in the intensity difference field outside the cavity, defined by

$$
S_{\mathrm{D}}(\Omega)=\frac{1}{S_{0}} \int_{-\infty}^{+\infty} \mathrm{d} \tau \mathrm{e}^{-i \Omega \tau}\left\langle\left(\hat{I}_{1}(t+\tau)-\hat{I}_{2}(t+\tau)\right)\left(\hat{I}_{1}(t)-\hat{I}_{2}(t)\right)\right\rangle_{\mathrm{ss}},
$$

where the shot noise from both beams is $S_{0}=2 \gamma_{\text {out }}\left(\left\langle I_{1}\right\rangle+\left\langle I_{2}\right\rangle\right)$. Classical optics prescribes $S_{\mathrm{D}}(\Omega) \geq 1$. In Fig. 2 we plot $S_{\mathrm{D}}(\Omega)$ for the same set of parameters as in Fig. 1 . A noticeable noise suppression around $\Omega=0$ is observed, clear evidence of a non-classical regime.

In conclusion we have presented a microscopic theory of HRS in semiconductor microcavities, we have calculated emission spectra as a function of excitation energy, detuning, and angles, and calculated the output spectrum of fluctuations in the intensity difference field which shows a noticeable non-classical behavior. We hope our results stimulate experimental work on cavity HRS.

\section{References}

[1] For a review, see P. R. Berman (Ed.), Cavity Quantum Electrodynamics, Academic Press, Boston 1994.

[2] C. Weisbuch, M. Nishioka, A. Ishikawa, and Y. Arakawa, Phys. Rev. Lett. 69, 3314 (1992).

[3] T. B. Norris, J. K. Rhee, C. Y. Sung, Y. Arakawa, M. Nishioka, and C. Weisbuch, Phys. Rev. B 50, 14663 (1984).

[4] Vu Duy Phach, A. Bivas, B. Hönerlage, and J. B. Grun, phys. stat. sol. (b) 86, 159 (1978).

[5] B. Hönerlage, A. Bivas, and Vu Duy Phach, Phys. Rev. Lett. 41, 49 (1978).

[6] For review, see B. Hönerlage, R. Levy, J. B. Grun, C. Klingshirn, and K. Bohnert, Phys. Rep. 124, 161 (1985).

[7] S. Savasta and R. Girlanda, Phys. Rev. Lett. 77, 4736 (1996).

[8] S. Savasta and R. Girlanda, submitted for publication.

[9] M. Combescot and R. Combescot, Phys. Rev. Lett. 61, 117 (1988).

[10] Th. Östreich, K. Schönhammer, and L. J. Sham, Phys. Rev. Lett. 74, 4698 (1995).

[11] V. M. Axt and A. Stahl, Z. Phys. B 93, 195 (1994).

[12] K. Victor, V. M. Aхт, and A. Stahl, Phys. Rev. B 51, 14164 (1995).

[13] P. N. Butcher and D. Cotter, The Elements of Nonlinear Optics, Cambridge University Press, 1990.

[14] For review, see W. H. Louisell, Quantum Statistical Properties of Radiation, Wiley, New York 1973.

H. Haken, Rev. Mod. Phys. 47, 67 (1975).

[15] M. G. Raymer and J. Mostowski, Phys. Rev. A 24, 1980 (1981).

[16] H. J. Carmichael, R. J. Brecha, M. G. Raizen, and H. J. Kimble, Phys. Rev. A 40, 5516 (1989). 
